\title{
Kallikrein-related peptidases in triple negative breast cancer: prognostic value of quantitatively assessed KLK8, KLK10 and KLK11 mRNA expression
}

\section{Yueyang Liu}

Klinikum rechts der Isar der Technischen Universität München: Klinikum rechts der Isar der Technischen Universitat Munchen

\section{Sarah Preis}

Klinikum rechts der Isar der Technischen Universität München: Klinikum rechts der Isar der Technischen Universitat Munchen

\section{Geng Xiaocong}

Klinikum rechts der Isar der Technischen Universität München: Klinikum rechts der Isar der Technischen Universitat Munchen

\section{Weiwei Gong}

Klinikum rechts der Isar der Technischen Universität München: Klinikum rechts der Isar der Technischen Universitat Munchen

\section{Marion Kiechle}

Klinikum rechts der Isar der Technischen Universität München: Klinikum rechts der Isar der Technischen Universitat Munchen

\section{Tobias Dreyer}

Klinikum rechts der Isar der Technischen Universität München: Klinikum rechts der Isar der Technischen Universitat Munchen

\section{Viktor Magdolen}

Klinikum rechts der Isar der Technischen Universität München: Klinikum rechts der Isar der Technischen Universitat Munchen

\section{Julia Dorn ( $\nabla$ juliadorn@web.de)}

Klinikum rechts der Isar der Technischen Universität München: Klinikum rechts der Isar der Technischen Universitat Munchen https://orcid.org/0000-0002-4349-9716

\section{Research Article}

Keywords: KLK8, KLK10, KLK11, triple-negative breast cancer, TNBC, mRNA expression 
DOI: https://doi.org/10.21203/rs.3.rs-365110/v1

License: (c) (1) This work is licensed under a Creative Commons Attribution 4.0 International License. Read Full License 


\section{Abstract}

Background. Triple-negative breast cancer (TNBC) is a breast cancer subtype with poor prognosis and limited targeted therapy options. Multiple KLKs have been described to play key roles in carcinogenesis and metastasis of breast cancer.

Purpose. In the present study, the clinical significance of KLK8, KLK10, and KLK11 mRNA expression in tumor tissue of TNBC patients was investigated.

Methods. The mRNA expression levels of KLK8, 10, and 11 were quantified by quantitative PCR and their prognostic values were analyzed in a large, well-characterized TNBC cohort $(n=123)$.

Results. Significantly positive correlations were observed between all three KLK mRNA levels indicating coordinate expression of these proteases in TNBC. In univariate analyses, both elevated KLK8, KLK10 as well as all combinations of the three factors (KLK8 + KLK10, KLK8 + KLK11, KLK10 + KLK11, KLK8 + KLK10 + KLK11) were significantly associated with shortened disease-free survival (DFS), while high mRNA levels of KLK11, as well as KLK10 + KLK11 were significantly associated with shortened overall survival (OS). In multivariate Cox regression analyses, KLK10 and all combined factors remained unfavorable independent predictive markers for DFS, while high KLK11 mRNA expression represented an unfavorable independent predictor for OS.

Conclusions. Increased KLK8, KLK10, and KLK11 mRNA expression levels are associated with unfavorable prognosis in triple-negative breast cancer. The combination of KLK8 + KLK10 + KLK11 may represent a stronger prognostic biomarker for DFS than KLK8, KLK10, KLK11 alone, or other combinations thereof, whereas KLK11 mRNA expression is an independent prognostic biomarker for OS in TNBC patients.

\section{Background}

Triple-negative breast cancer (TNBC) is characterized by the lack of estrogen receptor (ER) and progesterone receptor (PR) expression as well as the absence of human epidermal growth factor receptor 2 (HER2) amplification. TNBC accounts for about $17 \%$ of breast cancers and is associated with a more aggressive course and a worse prognosis of the disease, compared to hormone-related breast cancer [1]. TNBC occurs more commonly in younger women ( $<50$ years), and more likely shows distant recurrence. TNBC tends to disseminate to vital organs like the brain and lungs, leading to a median survival time of fewer than 13 months in metastatic TNBC, compared to other subtypes of breast cancer which preferentially metastasize to the bones [2-4]. Furthermore, the molecular features of TNBC exclude these patients from available targeted, anti-hormonal or HER2-targeting therapies.

As approximately $20 \%$ of TNBC patients show a BRCA $1 / 2$ mutation, genetic testing for all TNBC patients diagnosed at the age of 60 years or younger is recommended. For BRCA1/2 mutation carriers, treatment with PARP inhibitors seems to be a promising therapeutic tool [5]. Apart from this, few additional 
systemic chemotherapies (like platinum- or taxane-containing chemotherapy regimens or post adjuvant capecitabine therapy) have been introduced into the management of TNBC, with only minor changes in outcome for the price of higher burden in toxicity $[1,5,6]$. Therefore, valid biomarkers for the development of individualized treatment and improvment of TNBC management are urgently needed.

The kallikrein-related peptidase (KLK) family encompasses 15 closely related serine proteases, which are encoded by the genes $K L K 1-15$ located on the long arm of chromosome 19q13.4 [7]. Accumulating evidence has revealed that KLKs are dysregulated and implicated in tumor-relevant processes in various hormone-dependent malignancies like ovarian, breast and prostate cancer [8, 9]. In breast cancer, KLK310 and 12-15 but not KLK11 have been reported to serve as diagnostic and prognostic markers [10-13].

KLK8, a serine protease with trypsin-like activity, has been described to be elevated in several malignancies like lung, colon, cervical, and ovarian cancer. It was reported to efficiently cleave ECMrelated proteins like casein, fibronectin, and gelatin, thus promoting proteolysis in the pericellular space of cancer cells and potentially facilitating invasion of tumors [14-18]. Still, in ovarian cancer, elevated KLK8 expression was suggested to be a favorable prognostic marker for prolonged disease-free and overall survival. Additionally, tumors with increased KLK8 concentrations were diagnosed with lower grading and reduced residual tumor mass after surgery [19]. Moreover, overexpression of KLK8 in non small lung cancer cell lines suppresses cell invasion and tends to be a positive predictor for a prolonged tumor-free survival as well [20].

In breast cancer, KLK8 is downregulated on the mRNA level, compared to non-tumorous tissue. Still, high KLK8 mRNA expression in breast tumors was associated with advanced TNM stages, positive nodal status and poor prognosis [21]. Particularly, Michaelidou et al. [13] also showed KLK8 mRNA expression levels to be significantly higher in TNBC than in other subtypes of breast cancer.

KLK10 is widely distributed in various human organs and biological fluids and is broadly involved in pathophysiological processes [21]. Cumulative evidence indicates that KLK10 represents an unfavorable prognostic marker in colon, renal clear cell, colorectal and gastric cancer [22-24], but contrariwise KLK10 has also been associated with tumor-suppression in prostate and testicular cancer by modulation of proliferation and apoptosis. Moreover, KLK10 has been suggested to be a favourable biomarker, e.g. in ovarian cancer [25-27].

Similar to KLK8, also KLK10 mRNA as well as antigen expression levels were found to be downregulated in breast cancerous tissue, compared to non-cancerous breast tissue [28, 29]. KLK10 has been identified to be an important factor in tamoxifen and trastuzumab resistance, thus representing a possible therapeutic target [30,31].

KLK11 seems to be an important enzyme for several physiological processes in humans and is expressed in the tissue of the esophagus, salivary gland, skin, vagina, and cervix [32]. Besides that, it has been described as cancer biomarker: Geng et al. $[25,33]$ reported that both high KLK11 mRNA and protein levels are significantly associated with a favorable prognosis, revealing that KLK11 may exert an anti- 
tumorigenic role in ovarian cancer. KLK11 expression levels are higher in low grade breast cancer tissues and a loss of KLK11 was described as the malignancy increases [34]. However, the role of KLK11 as a biomarker in breast cancer has not yet been explored.

Besides the individual effects of the KLKs, it is known that these proteases are often parts of proteolytic networks and can be coordinately expressed and regulated. It was shown before that KLK8, KLK10 and KLK11 are expressed in breast tissue [35]. Together with the close proximity of these genes within the kallikrein locus [36] and the absence of individual classical promoter regions for each kallikrein [37], this raises the possibility for coordinate expression of these genes.

Altogether, KLK8, KLK10, and KLK11 may have the potential to serve as prognostic biomarkers and represent possible targets for therapy in breast cancer. The present study was designed to further explore the hypothesis, that KLK8, KLK10, and KLK11 are co-regulated in TNBC and that mRNA expression levels of these KLKs or their combinations are prognostic biomarkers.

\section{Methods}

\section{Patients}

One-hundred and twenty-three patients afflicted with triple-negative breast cancer (TNBC, $n=123$ ) were enrolled in the study conducted between the years 1988 and 2012 at the Department of Obstetrics and Gynecology, Klinikum rechts der Isar, Technical University of Munich (TUM). Tumor tissues were collected after inspection by pathologists and immediately stored in liquid nitrogen. Tumors were routinely tested for ER, PR, and HER2 expression and categorized as triple-negative breast cancer, if there was a lack of estrogen receptor (ER) and progesterone receptor (PR) protein expression and absence of or low HER2 expression (immunohistochemically determined score $0,1+$; or score $2+$ with negative fluorescence in situ hybridizations [FISH] test ) [38].

All patients primarily underwent standard surgical procedures including mastectomy or breast conserving surgery and none of them had distant metastasis at the time of surgery. Invasive ductal carcinoma subtype accounted for $87 \%$ (109/123) and the rest were more rare subtypes like medullary, lobular and others. Regarding treatment, adjuvant therapy was administered based on consensus recommendations at that time. $72 \%(89 / 123)$ of the patients were treated with anthracycline- or cyclophosphamide-based chemotherapy. The biomarker study in breast tumor tissues was approved by the local Ethics Committee (TU Munich, No. 491/17 S), and performed in accordance with the Declaration of Helsinki. Written informed consent of the study was available for all patients.

\section{Real-time polymerase chain reaction}

Universal ProbeLibray probes (Roche) were used in the quantitative polymerase chain reaction (qPCR) (for details see [14].. Gene-specific primers were designed with the Universal Probe Library Assay Design 
Center software (https://lifescience.roche.com). The following primers (Metabion) and hydrolysis probes from the Universal Probe Library (Roche) were used:

KLK8 (NM_007196) [14]:

Forward: 5'- CAGCAAAGGGGCTGACAC-3' (782-799)区

Reverse: 5'- GACCTCCCACAGGGGTCT-3' (869-886)凹

KLK8-probe: 5'-FAM- TGCCCTGG -3'-dark quencher; amplicon size: 105 bp.

The assay detects the KLK8 mRNA transcript variant 2, encoding the canonical KLK8 protein.

KLK10 (NM_002776.4, NM_145888.2, and NM_001077500.1) [33]:

Forward: 5'-CAGGTCTCGCTCTTCAACG-3' (397-415, 259-277, 298-316)凹

Reverse: 5'-GAGCCCACAGTGGCTTGT-3' (485-502, 347-364, 386-403)『

KLK10-probe: 5'-FAM-TCCACTGC-3'-dark quencher; amplicon size: 106 bp.

The assay detects the three major KLK10 mRNA transcript variants 1, 2 and 3, all encoding full-length KLK10.

KLK11 (NM_006853.2, NM_144947.1) [33]:

Forward: 5'-GCTTGCTCTGGCAACAGG-3' (150-167, 232-249)》

Reverse: 5'-AGTGAGGCTTGCACTCGAAC-3' (201-220, 283-302)区

KLK11-probe: 5'-FAM-GAGACCAG-3'-dark quencher; amplicon size: 71 bp.

The assay detects the KLK11 mRNA transcript variants 1 and 2, which encode full-length KLK11.

HPRT1 (NM_000194) [33]:

Forward: 5'-TGACCTTGATTTATTTTGCATACC-3' (218-241)凶

Reverse: 5'-CGAGCAAGACGTTCAGTCCT-3' (300-319)区

HPRT1-probe: 5'-FAM-GCTGAGGA-3'-dark quencher; amplicon size: 102 bp.

The assay detects the HPRT1 mRNA NM_000194, encoding full-length HPRT1.

Due to fact that amplification efficiencies vary among different qPCR assays, dilution series were employed to determine the differences concerning efficiency between the assays for the target genes and the housekeeping gene HPRT1 [39]. Except for KLK10, the amplification efficiencies of KLK8 and KLK11 
approximated that of HPRT. As described previously, the $2 \Delta \Delta$ Ct method was applied for the calculation of relative KLK8 and KLK11 mRNA expression [14]. As to KLK10, relative KLK10 mRNA expression was quantitatively accessed using the efficiency correction method [33].

\section{Statistics}

The correlation of mRNA expression levels of KLKs with clinicopathological parameters of the patients was assessed by the Chi-square test. Survival analyses were performed by constructing Kaplan-Meier curves. The log-rank test is proposed to evaluate group differences in the survival functions. Associations of KLKs and clinical parameters with patients' survival were additionally determined by univariate and multivariable Cox regression analysis and expressed as hazard ratio (HR) as well as its $95 \%$ confidence interval $(95 \% \mathrm{Cl})$. The correlations between continuous variables of KLKs were examined with the MannWhitney $U$ test and the Spearman rank correlation $\left(r_{s}\right)$. Box plots were drawn to indicate differences. All calculations were performed with the SPSS statistical analysis software (version 20.0; SPSS Inc., Chicago, IL, USA). P values of less than 0.05 were considered statistically significant. For further details, see $[14 ; 33]$.

\section{Results}

\section{KLK8, 10, and 11 mRNA expression in tumor tissues of triple-negative breast cancer (TNBC) patients and their correlation with clinical parameters}

KLK8, 10, and 11 mRNA expression levels were quantified by established qPCR assays $[25,33]$ in 123 cases of triple-negative breast cancer tissues. KLK8 mRNA levels ranged from 0.00 to 19.34 (median: 0.21 ), KLK10 mRNA expression ranged from 0.00 to 10.53 (median: 0.15), and KLK11 mRNA expression ranged from 0.00 to 2.70 (median: 0.01, a supplementary figure file shows this in more detail [see Suppl. file 1]).

mRNA expression of KLK8 and KLK11 was categorized by the $50^{\text {th }}$ percentile (median) into a low-expressing versus a high-expressing group, and KLK10 by the $67^{\text {th }}$ percentile in a low expression group (tertiles $1+2$ ) versus a high expression group (tertile 3 ).

By applying Spearman correlation analysis, a strong positive correlation was observed between KLK8 and KLK10 ( $\left.r_{s}=0.612, p<0.001\right)$ as well as between KLK10 and KLK11 ( $r_{s}$ $=0.722, \mathrm{p}<0.001)$, whereas the correlation between KLK8 and KLK11 mRNA was less pronounced $\left(r_{s}=0.460, p<0.001\right.$; an additional figure file shows this in more detail [see Suppl. file 2]).

The associations between low and high expression groups of the three KLK pairs were also validated by box plot analysis (Mann-Whitney test; p-value in all cases $<0.001$, Fig. 1) indicating co-regulation of the three KLK genes in TNBC. Due to this observation, we 
further dichotomized the mRNA levels into either a KLK8+KLK10, KLK8+KLK11, KLK10+KLK11, or KLK8+KLK10+KLK11 low-expressing group (mRNA levels below the respective cutoff value) versus high-expressing groups (KLK8 and/or KLK10 and/or KLK11 mRNA levels above the respective cutoff value) for statistical analyses.

The associations between relative KLK mRNA expression levels and the established clinical variables, including age, lymph node status, and tumor size, are summarized in Table 1. Neither KLK8 nor KLK10 nor KLK11 mRNA expression levels were associated with clinicopathological parameters of TNBC. Similarly, no statistically significant associations were observed between KLK combinations and clinical parameters (see Suppl. Table 1).

Table 1 Association between KLKs mRNA expression levels and clinicopathological parameters in patients with triple-negative breast cancer

\begin{tabular}{llll}
\hline Clinicopathological parameters & KLK8 $^{\mathrm{a}}$ & $\mathrm{KLK10}$ & $\mathrm{KLK11}^{\mathrm{a}}$ \\
& low/high & low/high & low/high \\
\hline Age & $\mathrm{p}=0.907$ & $\mathrm{p}=0.294$ & $\mathrm{p}=0.761$ \\
$\leq 60$ years & $31 / 30$ & $43 / 25$ & $33 / 28$ \\
$>$ 60 years & $27 / 25$ & $39 / 15$ & $23 / 22$ \\
\hline Lymph node status & $\mathrm{p}=0.756$ & $\mathrm{p}=0.990$ & $\mathrm{p}=0.473$ \\
\hline N0 & $31 / 31$ & $45 / 22$ & $33 / 26$ \\
\hline N1/N2/N3 & $27 / 24$ & $37 / 18$ & $23 / 24$ \\
\hline Tumor Size & $\mathrm{p}=0.843$ & $\mathrm{p}=0.207$ & $\mathrm{p}=0.665$ \\
\hline$\leq 20$ mm & $16 / 14$ & $25 / 8$ & $15 / 15$ \\
\hline$>20$ mm & $42 / 40$ & $56 / 32$ & $41 / 34$ \\
\hline
\end{tabular}

a $C h i-s q u a r e ~ t e s t ~\left(c u t-o f f\right.$ point: $K L K 8=50^{\text {th }}$ percentile, KLK10 $=67^{\text {th }}$ percentile, KLK1 $1=50^{\text {th }}$ percentile).

Due to missing values, numbers do not always add up to $\mathrm{n}=123$.

\section{Association of KLK8, 10, and 11 mRNA expression levels with disease-free survival (DFS) and overall survival (OS) in univariate Cox regression analysis}

Univariate Cox regression analysis was performed to evaluate the prognostic relevance of KLKs, the combination thereof, and the clinicopathological parameters regarding the 
patient outcome in the TNBC cohort (Table 2). Among the clinical variables, advanced age and a positive lymph node status indicated a significantly shorter DFS and OS.

Table 2 Univariate Cox regression analysis of clinical outcome in triple-negative breast cancer for tumor biological factors. 


\begin{tabular}{|c|c|c|c|c|c|c|}
\hline \multirow{3}{*}{$\begin{array}{l}\text { Clinicopathological } \\
\text { parameters }\end{array}$} & \multirow{2}{*}{\multicolumn{3}{|c|}{$\begin{array}{l}\text { DFS } \\
\text { DFS }\end{array}$}} & \multirow{2}{*}{\multicolumn{3}{|c|}{$\begin{array}{l}\text { OS } \\
\text { OS }\end{array}$}} \\
\hline & & & & & & \\
\hline & $\mathrm{No}^{\mathrm{a}}$ & HR $(95 \% \mathrm{CI})^{\mathrm{b}}$ & $\mathbf{p}$ & $\mathrm{No}^{\mathrm{a}}$ & HR $(95 \% \mathrm{CI})^{\mathrm{b}}$ & p \\
\hline Age & & & 0.005 & & & $<0.001$ \\
\hline$\leq 60$ years & 65 & 1 & & 65 & 1 & \\
\hline$>60$ years & 52 & $2.09(1.25-3.52)$ & & 56 & $2.76(1.62-4.70)$ & \\
\hline Lymph node status & & & 0.023 & & & 0.018 \\
\hline No & 63 & 1 & & 65 & 1 & \\
\hline $\mathrm{N} 1 / \mathrm{N} 2 / \mathrm{N} 3$ & 54 & $1.82(1.09-3.05)$ & & 56 & $1.86(1.11-3.10)$ & \\
\hline Tumor Size & & & 0.058 & & & 0.076 \\
\hline$\leq 20 \mathrm{~mm}$ & 32 & 1 & & 33 & 1 & \\
\hline$>20 \mathrm{~mm}$ & 84 & $1.89(0.98-3.64)$ & & 87 & $1.85(0.94-3.66)$ & \\
\hline KLK8 mRNA $^{c}$ & & & 0.037 & & & 0.313 \\
\hline low & 54 & 1 & & 57 & 1 & \\
\hline high & 54 & $1.88(1.04-3.41)$ & & 54 & $1.37(0.74-2.52)$ & \\
\hline $\mathrm{KLK} 10$ mRNA $^{\mathrm{c}}$ & & & 0.045 & & & 0.076 \\
\hline low & 78 & 1 & & 81 & 1 & \\
\hline high & 37 & $1.78(1.01-3.12)$ & & 38 & $1.68(0.95-2.99)$ & \\
\hline KLK11 mRNA ${ }^{\mathrm{c}}$ & & & 0.053 & & & 0.016 \\
\hline low & 52 & 1 & & 54 & 1 & \\
\hline high & 50 & $1.85(0.99-3.44)$ & & 50 & $2.29(1.17-4.49)$ & \\
\hline KLK8+KLK10 d & & & 0.013 & & & 0.164 \\
\hline low & 47 & 1 & & 50 & 1 & \\
\hline high & 60 & $2.28(1.19-4.34)$ & & 60 & $1.57(0.83-2.95)$ & \\
\hline KLK10+KLK11 & & & 0.046 & & & 0.035 \\
\hline low & 46 & 1 & & 48 & 1 & \\
\hline high & 54 & $1.95(1.01-3.78)$ & & 54 & $2.12(1.05-4.28)$ & \\
\hline $\mathrm{KLK} 8+\mathrm{KLK} 11^{\mathrm{f}}$ & & & 0.042 & & & 0.147 \\
\hline low & 31 & 1 & & 33 & 1 & \\
\hline high & 66 & $2.18(1.03-4.61)$ & & 66 & $1.76(0.82-3.79)$ & \\
\hline KLK8+KLK10+KLK11g & & & 0.019 & & & 0.106 \\
\hline low & 27 & 1 & & 29 & 1 & \\
\hline high & 69 & $2.85(1.19-6.86)$ & & 69 & $1.99(0.86-4.59)$ & \\
\hline
\end{tabular}

Chi-square test, significant p-values $(\mathrm{p}<0.05)$ are indicated in bold, trends towards significance $(\mathrm{p}<0.08)$ in italics.

a Number of patients.

b HR: hazard ratio (CI: confidence interval) of univariate Cox regression analysis. 
c Dichotomized into low and high levels by the $50^{\text {th }}$ percentile for KLK8 and KLK11, by $67^{\text {th }}$ percentile for KLK10.

d Dichotomized into low level by KLK8 low and KLK10 low, and high level by KLK8 high and/or KLK10 high.

e Dichotomized into low level by KLK10 low and KLK11 low, and high level by KLK10 high and/or KLK11 high.

$\mathrm{f}$ Dichotomized into low level by KLK8 low and KLK11 low, and high level by KLK8 high and/or KLK11 high.

g Dichotomized into low level by KLK8 low, KLK10 low as well as KLK11 low, and high level by KLK8 high, KLK10 high and/or KLK11 high.

Due to missing values, numbers do not always add up to $n=117$ (DFS) and $n=121$ (OS).

Elevated KLK8 mRNA expression levels were found to be a significant predictive factor for worse DFS ( $\mathrm{HR}=1.88, \mathrm{p}=0.037$ ), but not for OS, indicating an about two-fold increased probability of tumor progression in the KLK8 high-expressing group. KLK10 mRNA levels also represented a significant predictive marker for shortened DFS (HR, 1.78; $p=0.045$ ), but only showed a trend towards significance for OS (HR: 1.68; $\mathrm{p}=0.076$ ). Regarding KLK11, high expression levels showed a trend towards significance for DFS (HR: 1.85; $\mathrm{p}=$ 0.053 ) only, but were notably associated with worse OS (HR: $2.29 ; \mathrm{p}=0.016$ ).

KLK8+KLK10 high expression, as well as KLK8+KLK11 high expression were also significantly associated with an elevated probability of disease recurrence (HR: 2.28, p = 0.013 and HR: 2.18, $\mathrm{p}=0.042$ ), while KLK10+KLK11 high expression was significantly not only correlated with shorter DFS (HR: $1.95, \mathrm{p}=0.046$ ) but also with shorter OS (HR: 2.12, $\mathrm{p}=0.035)$. Compared to the KLKs alone, the combined paired factors values, thus, could mildly increase the predictive power for DFS and/or OS. Finally, combination of all three biological factors (KLK8+KLK10+KLK11 low/low/low versus high and/or high and/or high) revealed that patients displaying tumor tissue-associated low expression of all three factors had a 2.85-fold reduced risk for disease recurrence compared to the group with high expression of at least one of these KLKs.

The impact of these factors on patient survival was also confirmed by Kaplan-Meier estimation. As shown by the respective survival curves, high KLK8 (Fig. 2A) and high KLK10 levels (Fig. 2B) were significantly associated with shortened DFS $(p=0.033, p=$ 0.042, respectively), whereas high KLK11 expression (Fig. 2C) was notably associated with both worse DFS $(p=0.049)$ and OS $(p=0.013)$. In case of the combined factors, all combinations represented prognostic factors for DFS (Fig. 3), whereas only the combination KLK10+KLK11 displayed a significant association with OS (see Table 2). 
Association of KLK8, 10 and 11 mRNA expression levels with disease-free survival (DFS) and overall survival (OS) in multivariable Cox regression analysis

The independence of KLKs as prognostic factors in TNBC was studied in multivariable Cox regression analysis (Table 3 ). We established a base model containing age, lymph node status, and tumor size. Here, age was the only clinical parameter displaying predictive power for DFS (HR, 2.12; $\mathrm{p}=0.025)$ and OS $(\mathrm{HR}, 3.18 ; \mathrm{p}=0.002)$. Among the tumor biological factors (added separately to the base model), KLK8 mRNA expression lost its prognostic significance for DFS, while KLK10 mRNA expression significantly contributed to the base model for DFS (HR: 2.19, $\mathrm{p}=0.019$ ). Elevated KLK11 mRNA expression turned out to represent an independent unfavorable predictor of OS (HR, 2.06; $p=0.044)$.

Table 3 Multivariate Cox regression analysis of clinical outcome in triple-negative breast cancer for tumor biological factors. 


\begin{tabular}{|c|c|c|c|c|c|c|}
\hline \multirow[t]{2}{*}{ Clinicopathological parameters } & \multicolumn{3}{|c|}{ DFS } & \multicolumn{3}{|c|}{ OS } \\
\hline & $\mathrm{No}^{\mathrm{a}}$ & HR $(95 \% \mathrm{CI})^{b}$ & $\mathbf{p}$ & $\mathrm{No}^{\mathrm{a}}$ & HR $(95 \% \text { CI) })^{b}$ & $\mathrm{p}$ \\
\hline Age & & & 0.025 & & & 0.002 \\
\hline$\leq 60$ years & 54 & 1 & & 54 & 1 & \\
\hline$>60$ years & 41 & $2.12(1.10-4.09)$ & & 43 & $3.18(1.53-6.59)$ & \\
\hline Lymph node status & & & 0.236 & & & 0.286 \\
\hline No & 51 & 1 & & 53 & 1 & \\
\hline N1/N2/N3 & 44 & $1.49(0.77-2.87)$ & & 44 & $1.46(0.73-2.94)$ & \\
\hline Tumor Size & & & 0.305 & & & 0.423 \\
\hline$\leq 20 \mathrm{~mm}$ & 28 & 1 & & 29 & 1 & \\
\hline$>20 \mathrm{~mm}$ & 67 & $1.51(0.69-3.33)$ & & 68 & $1.41(0.61-3.29)$ & \\
\hline KLK8 mRNA ${ }^{\mathrm{C}}$ & & & 0.062 & & & 0.390 \\
\hline low & 46 & 1 & & 48 & 1 & \\
\hline high & 49 & $1.90(0.97-3.72)$ & & 49 & $1.36(0.68-2.71)$ & \\
\hline KLK10 mRNA ${ }^{C}$ & & & 0.019 & & & 0.058 \\
\hline low & 63 & 1 & & 65 & 1 & \\
\hline high & 32 & $2.19(1.14-4.20)$ & & 32 & $1.95(0.98-3.91)$ & \\
\hline $\mathrm{KLK}_{11}$ mRNA $^{\mathrm{C}}$ & & & 0.113 & & & 0.044 \\
\hline low & 51 & 1 & & 53 & 1 & \\
\hline high & 44 & $1.70(0.88-3.26)$ & & 44 & $2.06(1.02-4.14)$ & \\
\hline $\mathrm{KLK} 8+\mathrm{KLK} 10^{\mathrm{C}}$ & & & 0.013 & & & 0.163 \\
\hline low & 39 & 1 & & 41 & 1 & \\
\hline high & 56 & $2.62(1.23-5.58)$ & & 56 & $1.70(0.81-3.59)$ & \\
\hline KLK10+KLK11 C & & & 0.049 & & & 0.054 \\
\hline low & 45 & 1 & & 47 & 1 & \\
\hline high & 50 & $1.97(1.00-3.86)$ & & 50 & $2.03(0.99-4.17)$ & \\
\hline KLK8+KLK11 ${ }^{\mathrm{C}}$ & & & 0.048 & & & 0.171 \\
\hline low & 31 & 1 & & 33 & 1 & \\
\hline high & 64 & $2.15(1.01-4.58)$ & & 64 & $1.72(0.79-3.71)$ & \\
\hline $\mathrm{KLK} 8+\mathrm{KLK} 0+\mathrm{KLK} 11^{\mathrm{C}}$ & & & 0.019 & & & 0.124 \\
\hline low & 27 & 1 & & 29 & 1 & \\
\hline high & 68 & $2.87(1.19-6.91)$ & & 68 & $1.93(0.83-4.48)$ & \\
\hline
\end{tabular}

Chi-square test, significant p-values $\overline{(p<0.05)}$ are indicated in bold, trends towards significance $(\mathrm{p}<0.08)$ in italics.

a Number of patients.

b HR: hazard ratio (CI: confidence interval) of univariate Cox regression analysis. 
C Dichotomization into a low versus high expressing group was performed as described in Table 2.

All analyzed combinations significantly contributed to the base model for DFS and thus represented independent predictive markers. Strikingly, both the low-groups of the combinations KLK8+KLK10 and KLK8+KLK10+KLK11, respectively, had an over 2.5-fold reduced risk for disease recurrence when compared to the high-groups (HR, 2.62; $\mathrm{p}=$ 0.013; HR: 2.87, $\mathrm{p}=0.019$; respectively).

\section{Discussion}

The members of the kallikrein-related peptidase family (KLKs) have been described to be coordinately expressed and to play a major role in different cancer entities including ovarian and breast cancer [14, 25, $28,33]$. In the present study, we analyzed mRNA expression levels of KLK8, KLK10 and KLK11 in a welldefined cohort of triple-negative breast cancer (TNBC), and further estimated their prognostic value.

When exploring the prognostic impact of KLK8, 10 and 11 in TNBC, both elevated KLK8 and KLK10 mRNA expression levels were shown to be significantly associated with shortened DFS while high expression levels of KLK11 were notably associated with worse OS. In multivariate Cox regression analysis, KLK8 mRNA expression lost its prognostic significance for DFS, while KLK10 mRNA expression significantly contributed to the base model for DFS.

In line with our findings, Michaelidou and co-workers [13] found that high KLK8 mRNA expression was notably correlated with poor prognosis and more aggressive subtypes of breast cancer, such as advanced TNM stages and positive nodal status. Particularly, they also showed that KLK8 mRNA expression levels were significantly higher in TNBC than other subtypes of this disease.

Likewise, the positive association between KLK10 levels and worse prognosis has been previously observed by Wang and co-workers [31] by analyzing mRNA data form more than four hundred HER2positive breast cancer patients from the TCGA database. Similarly, Luo and co-woekers [40] also investigated the correlation of elevated KLK10 antigen levels with higher risk of disease relapse and cancer-related death in tumor tissues of breast cancer patients. Altogether, more and more evidence is evolving regarding KLK8 and KLK10 to represent unfavorable predictive biomarkers in breast malignancy. For KLK11, there are only few data on it's prognostic impact in cancer, but it has been suggested to be a favorable prognostic marker e.g. in gastric, prostate, and ovarian cancer [41, 42]. Furthermore, high serum levels of KLK11 in ovarian cancer and prostate cancer patients may also be related to a favorable prognosis in these entities [44]. On the other hand, higher KLK11 levels seem to be markers of early disease in breast cancer and vanish during malignancy progression [34]. However, the prognostic value of KLK11 for breast cancer progression needs to be further validated. 
When analyzing the prognostic impact of KLK combinations (KLK8 + KLK10, KLK8 + KLK11, KLK10 + KLK11, KLK8 + KLK10 + KLK11), these biomarkers significantly contributed to the base model for DFS and thus represented independent predictive markers. This increase in predictive power for survival has already been described in ovarian cancer for the combination of KLK6 and 8 as well as KLK10 and KLK11 $[14,26]$. KLK8, KLK10, and KLK11 might be involved in synergistic mechanisms affecting tumor pathogenesis in TNBC. Hence, compared to KLKs alone, the combined paired factors allowed better identification of patients with unfavorable prognosis in this TNBC cohort.

There have been several studies to explore possible pathophysiological mechanisms including tumorigenicity of KLK8, KLK10 and KLK11. KLK8, abundantly expressed by highly reactive oligodendrocytes in the central nervous system, contributes to the degeneration of the spinal cord after injury and the modulation of neural plasticity development as well as demyelination [44, 45]. Patients with Alzheimer's disease expressed a 11.5 higher concentration of KLK8 compared to healtyh patients, which implicates KLK8 being part of the disease development of Alzheimer [46]. Moreover, KLK8 has been identified to be part of a proteolytic cascade targeting the skin barrier, thus being involved in dermal wound healing but also in pathological skin diseases, with high KLK8 levels correlating with disease severity [47]. Studies with patients suffering from bowel cancer show an effect of KLK8 in proliferation, invasion and migration in vitro. High KLK8 expression is significantly associated to an advanced, more aggressive disease and therefore to a shorter disease free and overall survival [48]. Regarding the ability of tumors to metastasize, KLK8 holds an important role in tumor invasion. The serine protease activity of KLK8 has an impact on fibronectin and collagen IV, which are both part of the extracellular matrix. Higher levels of fibronectin lead to tumor growth and metastases [49]. The loss of collagen IV results in a different tumor environment and increased tumor invasion in bowel cancer [50].

KLK10 is commonly supposed to promot tumor invation and metastasis via degrrading extracellular matrix proteins [51]. Furthermore, Li et al. [52] observed that KLK10 might lower the cisplatin-induced apoptosis, indicating that KLK10 might be coorelated with cisplatin resistance and serve as a potential therapeutic target in esophageal cancer cells. Moreover, KLK10 expression has been found to be significantly enhanced in established herceptin-resistant gastric cancer cell lines [53], in where the lower expression of KLK10 or suppressing PI3K/AKT pathway was able to decrease herceptin resistance and the overexpression showed the reverse effects, indicating that KLK10 displayed a key role in herceptin resistance by impacting the PI3K/AKT signaling pathway.

Possible mechanisms of the unfavorable biological role of KLK11 in malignancies have been explored as well. Xu et al. [54] found that a knockdown of KLK11 expression significantly inhibits growth and induces apoptosis of tumor cells in colorectal cancer cells in vitro. There, KLK11 inhibition activates the apoptosis signaling pathway by upregulating Bax expression and diminishing Bcl-2 expression. Moreover caspase-3 activity was induced, resulting in cancer cell death [54]. Furthermore, Zhang et al. [55] showed that the downregulation of KLK11 could inhibit the phosphatidylinositol-3-kinase (PI3K)/AKT pathway, which plays a critical role in tumor cell proliferation, invasion, and metastasis, thus affecting oxaliplatin resistance, suppressing cell growth and activating apoptosis in colorectal cancer cells [56]. Moreover, 
KLK11 cleaves insulin-like growth factor (IGF) binding protein 3 (IGFBP-3) and modulates functions of IGFs [34], which play important roles in the tumor growth and cell survival [57].

Various KLKs have been observed to be co-expressed and mostly downregulated when analyzing all subgroups of breast cancer tumors compared to non-malignant tissue, partly based on hormone-related regulatory mechanisms [58-62]. Androgens and estrogens are able to stimulate KLKs, especially KLK10 and KLK11 [58]. The expression levels of KLK10, 11, 14 in BT-474 breast cancer cells increase after stimulation with androgens [61]. 17 $\beta$-Estradiol leads to an increasion of KLK8 in hormone receptor expressing breast cancer cell lines MCF-7 and T-47D [61, 63]. Estrogens and androgens lead to an upregulation of KLK10 and KLK11 in breast cancer cell lines [63]. In the cervico-vaginal fluid, a peak of KLK11 is described in the secretory phase, suggesting a progestin stimulation of the KLKs [64], while KLK8 is highly expressed during the estrogen stimulated proliferative phase of the menstruation cycle [65]. This data has been supported by in vitro analysis of the KLK expression in 21 breast cancer cell lines (BCCLs). Here, an altered KLK translation was detected, mostly resulting in downregulation compared to non-malignant cells. However, significantly higher amounts of KLKs were detected in cells lacking the hormone receptors [60]. Apart from androgens and estrogens, glucocorticoids play an important role in the regulation of kallikrein-related peptidases. Dexamethason leads to an upregulation of KLK6, 8, 10 and a downregulation in $\mathrm{KLK5}, 6,8,10,11$ and 13 in different breast cancer cell lines [63]. Another possibly involved mechanism in the coordinate expression of different KLKs might be epigenetic regulation acquired during the course of the disease as well as dysregulated miRNA expression, escpecially in cancer progression [66].

In the current study, we indeed observed a pronounced, positive correlation between KLK10 and KLK11 mRNA expression levels in our homogenous TNBC cohort $\left(r_{s}=0.722\right)$, indicating that KLK10 and KLK11 are also co-expressed in this subtype of breast cancer. Additionally, moderate and/or strong positive relationships between KLK8 and KLK11 as well as between KLK8 and KLK10 mRNA expression levels in this cohort $\left(r_{s}=0.460, r_{s}=0.612\right.$, respectively) suggest coordinate expression of all KLKs analyzed in the subgroup of TNBC. At first sight, these results seem to be unexpected due to the fact that TNBC is characterized by the lack of the steroid hormone receptors ER and PR. However, it has been shown that the breast is an organ displaying prominent androgen-regulated expression [58]. In fact, also in breast cancer tissue, androgen receptor expression is frequently observed, with up to $50 \%$ of the cases in the TNBC subgroup [67]. Moreover, in androgen-receptor expressing breast cancer cell lines, KLK8, 10, and 11 and other KLKs are up-regulated by androgens both at the mRNA and the protein level [59]. Thus, it is tempting to speculate that KLK co-expression in TNBC is regulated, at least in part, by the androgensignaling pathway [58] .

The embedding of KLKs in a proteolytic network has been described for several biological compartments before, including the desquamation of the skin, liquefication of semen and prostate physiology [68]. These networks are dependent on the autocatalytic and cross-activation of KLKs like the activation of pro-KLK11 by KLK8 [69] and KLK11 [70], but also the bulk activation by other proteases such as MMP20 [71]. Up to now, one can still only speculate about the pathophysiological mechanisms of KLK8, KLK10, 
and KLK11 as biomarkers in cancer, and even more in TNBC. As we here showed that KLK8, KLK10, and KLK11 are valuable biomarkers in TNBC, further research should concentrate on possible ways of coordinate action of KLKs in TNBC and on the role of KLKs as a target for therapy.

\section{Conclusion}

In conclusion, for the first time, we quantified KLK8, KLK10 and KLK11 mRNA expression levels in a homogenous patient cohort of triple-negative breast cancer (TNBC). We show that the selected KLKs are coordinately expressed in TNBC tumor tissue. Elevated KLK8, KLK10, and KLK11 mRNA expression levels are unfavorable prognostic factors for patients with TNBC. Furthermore, the combined paired factor values increased the predictive power for DFS and/or OS compared to single factor analysis.

\section{Abbreviations}

$95 \% \mathrm{Cl}$

$95 \%$ confidence interval

DFS

disease-free survival

ER

estrogen receptor

$\mathrm{FISH}$

fluorescence in situ hybridizations

HER2

human epidermal growth factor receptor 2

HR

hazard ratio

IGF

insulin-like growth factor

IGFBP-3

insulin-like growth factor binding protein 3

KLK

kallikrein-related peptidase

OS

overall survival

$\mathrm{PI} 3 \mathrm{~K}$

phosphatidylinositol-3-kinase

PR

progesterone receptor

qPCR

quantitative real-time PCR 
TNBC

triple-negative breast cancer

\section{Declarations}

\section{Ethics approval and consent to participate}

The study was performed in accordance with the Declaration of Helsinki and approved by the local Ethics Committee (Faculty of Medicine, Technical University Munich, Ismaninger Str. 22, 81675 Muenchen, Germany, ethikkommission@mri.tum.de; project 491/17S). Written informed consent was obtained from all patients.

\section{Consent for publication}

Not applicable

\section{Availability of data and materials}

This article is original and has not been published elsewhere.

No sequencing data has been generated within this study.

Patient-related data are available via the Ethics Committee of the Medical Faculty of the Technical University of Munich, for researchers who meet the criteria for access to confidential data. Contact address: PD Dr. Julia Dorn, Klinische Forschergruppe der Frauenklinik, Ismaninger Str. 22, 81675 Munich, Germany; julia.dorn@tum.de. According to the Bavarian Data Protection Authority (BayLDA) and the General Data Protection Regulation (GDPR), patient-related data will only be made available to third parties after double-pseudonymization, undertaken by the Dept. of Medical Statistics and Epidemiology, Technical University of Munich.

\section{Competing interests}

The authors declare that they have no competing interests.

\section{Funding}

The present study was supported in part by a grant from the Deutsche Forschungsgemeinschaft, DO 1772/1-1 (JD), and from the Wilhelm Sander-Stiftung, contract number 2016.024.1 (TD). The funding sponsors had no role in the design of the study; in the collection, analyses, or interpretation of data, in the writing of the manuscript, and in the decision to publish the results.

\section{Authors' contributions}

YL and SP were responsible for acquisition of data, formal analysis, interpretation of data and drafting the manuscript. VM was responsible for conception and design, project administration, formal analysis, 
supervision and writing - review and editing. JD was responsible for conception and design, data curation, project administration, supervision, drafting the manuscript, review \& editing. TD was responsible for acquisition of data, formal analysis, interpretation of data, and review \& editing. XG, WG and MK were responsible for acquisition of data and writing - review \& editing. All authors approved the final manuscript version.

\section{Acknowledgements}

Not applicable

\section{References}

1. Lebert JM, Lester R, Powell E, Seal M, McCarthy J (2018) Advances in the systemic treatment of triple-negative breast cancer. Curr Oncol 25:S142-S150. DOI:10.3747/co.25.3954

2. Foulkes WD, Smith IE, Reis-Filho JS. Triple-negative breast cancer. N Engl J Med. 2010; 363:1938-48, DOI: 10.1056/NEJMra1001389

3. Kassam F, Enright K, Dent R, Dranitsaris G, Myers J, Flynn C et al (2009) Survival outcomes for patients with metastatic triple-negative breast cancer: implications for clinical practice and trial design. Clin Breast Cancer 9:29-33. DOI:10.3816/CBC.2009.n.005

4. Reis-Filho JS, Tutt AN (2008) Triple negative tumours: a critical review. Histopathology 52:108-118. DOI:10.1111/j.1365-2559.2007.02889.x

5. Robson M, Im SA, Senkus E, Xu B, Domchek SM, Masuda N et al (2017) Olaparib for Metastatic Breast Cancer in Patients with a Germline BRCA Mutation. N Engl J Med 377:523-533. DOI:10.1056/NEJMoa1706450

6. Palma G, Frasci G, Chirico A, Esposito E, Siani C, Saturnino C et al (2015) Triple negative breast cancer: looking for the missing link between biology and treatments. Oncotarget 6:26560-26574. DOI:10.18632/oncotarget.5306

7. Borgoño CA, Diamandis EP (2004) The emerging roles of human tissue kallikreins in cancer. Nat Rev Cancer 4:876-890. DOI:10.1038/nrc1474

8. Dorn J, Gkazepis A, Kotzsch M, Kremer M, Propping C, Mayer K et al (2014) Clinical value of protein expression of kallikrein-related peptidase 7 (KLK7) in ovarian cancer. Biol Chem 395:95-107. DOI:10.1515/hsz-2013-0172

9. Planque C, Ainciburu M, Heuze-Vourc'h N, Regina S, de Monte M, Courty Y (2006) Expression of the human kallikrein genes 10 (KLK10) and 11 (KLK11) in cancerous and non-cancerous lung tissues. Biol Chem 387:783-788. DOI:10.1515/BC.2006.098

10. Figueroa CD, Molina L, Bhoola KD, Ehrenfeld P (2018) Overview of tissue kallikrein and kallikreinrelated peptidases in breast cancer. Biol Chem 399(9):937-957. DOl:10.1515/hsz-2018-0111

11. Gong W, Liu Y, Preis S, Geng X, Petit-Courty A, Kiechle M et al (2020) Prognostic value of kallikreinrelated peptidase 12 (KLK12) mRNA expression in triple-negative breast cancer patients. Mol Med 
26(1):19. DOI:10.1186/s10020-020-0145-7

12. Haritos C, Michaelidou K, Mavridis K, Missitzis I, Ardavanis A, Griniatsos J et al (2018) Kallikreinrelated peptidase 6 (KLK6) expression differentiates tumor subtypes and predicts clinical outcome in breast cancer patients. Clin Exp Med 2:203-213. DOI:10.1007/s10238-018-0487-4

13. Michaelidou K, Ardavanis A, Scorilas A (2015) Clinical relevance of the deregulated kallikrein-related peptidase 8 mRNA expression in breast cancer: a novel independent indicator of disease-free survival. Breast Cancer Res Treat 152(2):323-336. DOI:10.1007/s10549-015-3470-8

14. Ahmed N, Dorn J, Napieralski R, Drecoll E, Kotzsch M, Goettig P et al (2016) Clinical relevance of kallikrein-related peptidase 6 (KLK6) and 8 (KLK8) mRNA expression in advanced serous ovarian cancer. Biol Chem 397:1265-1276. DOI:10.1515/hsz-2016-0177

15. Cané S, Bignotti E, Bellone S, Palmieri M, De las Casas L, Roman JJ et al (2004) The novel serine protease tumor-associated differentially expressed gene-14 (KLK8/Neuropsin/Ovasin) is highly overexpressed in cervical cancer. Am J Obstet Gynecol 190:60-66. DOI:10.1016/j.ajog.2003.07.020

16. Planque C, Choi YH, Guyetant S, Heuze-Vourc'h N, Briollais L, Courty Y (2010) Alternative splicing variant of kallikrein-related peptidase 8 as an independent predictor of unfavorable prognosis in lung cancer. Clin Chem 56:987-997. DOI:10.1373/clinchem.2009.138917

17. Rajapakse S, Ogiwara K, Takano N, Moriyama A, Takahashi T (2005) Biochemical characterization of human kallikrein 8 and its possible involvement in the degradation of extracellular matrix proteins. FEBS Lett 579:6879-6884. DOI:10.1016/j.febslet.2005.11.039

18. Yousef GM, Borgono CA, Popalis C, Yacoub GM, Polymeris ME, Soosaipillai A et al (2004a) In-silico analysis of kallikrein gene expression in pancreatic and colon cancers. Anticancer Res 24:43-51

19. Magklara A, Scorilas A, Katsaros D, Massobrio M, Yousef GM, Fracchioli S et al (2001) The human KLK8 (Neuropsin/Ovasin) gene: Identification of two novel splice variants and its prognostic value in ovarian cancer. Clin Cancer Res 7(4):806-811

20. Sher YP, Chou CC, Chou RH, Wu HM, Chang WSW, Chen CH et al (2006) Human kallikrein 8 protease confers a favorable clinical outcome in non-small cell lung cancer by suppressing tumor cell invasiveness. Cancer Res 66(24):11763-11770. DOI:10.1158/0008-5472.CAN-06-3165

21. Luo LY, Grass L, Howarth DJ, Thibault P, Ong H, Diamandis EP (2001) Immunofluorometric assay of human kallikrein 10 and its identification in biological fluids and tissues. Clin Chem 47:237-246

22. Alexopoulou D, Scorilas A, Prezas P, Xynopoulos D, Arnogiannaki N, Talieri M. Expression and clinical evaluation of Kallikrein-related peptidase 10 (KLK10) gene in colon cancer. AACR. 2007: 67 Suppl 9: 1767

23. Feng B, Xu WB, Zheng MH, Ma JJ, Cai Q, Zhang Y et al (2006) Clinical significance of human kallikrein 10 gene expression in colorectal cancer and gastric cancer. J Gastroenterol Hepatol 21(10):1596-1603. DOI:10.1111/j.1440-1746.2006.04228.x

24. Tailor PD, Kodeboyina SK, Bai S, Patel N, Sharma S, Ratnani A et al (2018) Diagnostic and prognostic biomarker potential of kallikrein family genes in different cancer types. Oncotarget 9:17876-17888. DOI:10.18632/oncotarget.24947 
25. Geng X, Liu Y, Dreyer T, Bronger H, Drecoll E, Magdolen V et al (2018) Elevated tumor tissue protein expression levels of kallikrein-related peptidases KLK10 and KLK11 are associated with a better prognosis in advanced high-grade serous ovarian cancer patients. Am J Cancer Res 8:1856

26. Hu J, Lei H, Fei X, Liang S, Xu H, Qin D et al (2015) NES1/KLK10 gene represses proliferation, enhances apoptosis and down-regulates glucose metabolism of PC3 prostate cancer cells. Sci Rep 5:17426. DOI:10.1038/srep17426

27. Yunes MJ, Neuschatz AC, Bornstein LE, Naber SP, Band V, Wazer DE (2003) Loss of expression of the putative tumor suppressor NES1 gene in biopsy-proven ductal carcinoma in situ predicts for invasive carcinoma at definitive surgery. Int J Radiat Oncol Biol Phys 56:653-657. DOI:10.1016/s03603016(03)00068-3

28. Yousef GM, Yacoub GM, Polymeris ME, Popalis C, Soosaipillai A, Diamandis EP (2004) Kallikrein gene downregulation in breast cancer. Br J Cancer 90:167-172. DOI:10.1038/sj.bjc.6601451

29. Dhar S, Bhargava R, Yunes M, Li B, Goyal J, Naber SP et al (2001) Analysis of normal epithelial cell specific-1 (NES1)/kallikrein 10 mRNA expression by in situ hybridization, a novel marker for breast cancer. Clin Cancer Res 7(11):3393-3398

30. Luo LY, Rajpert-De Meyts ER, Jung K, Diamandis EP (2001) Expression of the normal epithelial cellspecific 1 (NES1; KLK10) candidate tumour suppressor gene in normal and malignant testicular tissue. Br J Cancer 85:220-224. DOI:10.1054/bjoc.2001.1870

31. Wang Z, Ruan B, Jin Y, Zhang Y, Li J, Zhu L et al (2016) Identification of KLK10 as a therapeutic target to reverse trastuzumab resistance in breast cancer. Oncotarget 7:79494.

DOI:10.18632/oncotarget.13104

32. Loessner D, Goettig P, Preis S, Felber J, Bronger H, Clements JA et al (2018) Kallikrein-related peptidases represent attractive therapeutic targets for ovarian cancer. Expert Opin Ther Targets 9:745-763. DOI:10.1080/14728222.2018.1512587

33. Geng X, Liu Y, Diersch S, Kotzsch M, Grill S, Weichert W et al (2017) Clinical relevance of kallikreinrelated peptidase $9,10,11$, and 15 mRNA expression in advanced high-grade serous ovarian cancer. PLoS One 12:e0186847. DOI:10.1371/journal.pone.0186847

34. Sano A, Sangai T, Maeda H, Nakamura M, Hasebe T, Ochiai A (2007) Kallikrein 11 expressed in human breast cancer cells releases insulin-like growth factor through degradation of IGFBP-3. Int J Oncol 30:1493-1498

35. Shaw JL, Diamandis EP (2007) Distribution of 15 human kallikreins in tissues and biological fluids. Clin Chem 53(8):1423-1432. DOI:10.1373/clinchem.2007.088104

36. Yousef GM, Chang A, Scorilas A, Diamandis EP (2000) Genomic organization of the human kallikrein gene family on chromosome 19q13.3-q13.4. Biochem Biophys Res Commun 276:125-133.

DOI:10.1006/bbrc.2000.3448

37. Chow TF, Crow M, Earle T, El-Said H, Diamandis EP, Yousef GM (2008) Kallikreins as microRNA targets: an in silico and experimental-based analysis. Biol Chem 389(6):731-738.

DOI:10.1515/BC.2008.071

Page $21 / 27$ 
38. Yang F, Aubele M, Walch A, Gross E, Napieralski R, Zhao S et al (2017) Tissue kallikrein-related peptidase 4 (KLK4), a novel biomarker in triple-negative breast cancer. Biol Chem 398:1151-1164. DOI:10.1515/hsz-2017-0122

39. Bustin SA, Nolan T (2013) Analysis of mRNA expression by real-time PCR. In: Real-time PCR: advanced technologies and applications. Caister Academic Press, Norfolk, pp 51-88

40. Luo LY, Diamandis EP, Look MP, Soosaipillai AP, Foekens JA (2002) Higher expression of human kallikrein 10 in breast cancer tissue predicts tamoxifen resistance. $\mathrm{Br} J$ Cancer 86(11):1790-1796. DOI:10.1038/sj.bjc.6600323

41. Mavridis K, Scorilas A (2010) Prognostic value and biological role of the kallikrein-related peptidases in human malignancies. Future Oncol 6(2):269-285. DOI:10.2217/fon.09.149

42. Unal D, Tasdemir A, Oguz A, Eroglu C, Cihan YB, Turak EE et al (2013) Is human kallikrein-11 in gastric cancer treated with surgery and adjuvant chemoradiotherapy associated with survival? Pathol Res Pract 209(12):779-783. DOI:10.1016/j.prp.2013.09.004

43. Borgoño CA, Fracchioli S, Yousef GM, Rigault de la Longrais IA, Luo LY, Soosaipillai A et al (2003) Favorable prognostic value of tissue human kallikrein 11 (hK11) in patients with ovarian carcinoma. Int J Cancer 106(4):605-610. DOI:10.1002/ijc.11296

44. Terayama R, Bando Y, Takahashi T, Yoshida S (2004) Differential expression of neuropsin and protease $M /$ neurosin in oligodendrocytes after injury to the spinal cord. Glia 48:91-101. DOI:10.1002/glia.20058

45. Yoshida S (2003) Kallikrein-family serine protease in the central nervous system. Kaibogaku Zasshi 78:77-82

46. Shimizu-Okabe C, Yousef GM, Diamandis EP, Yoshida S, Shiosaka S, Fahnestock M (2001) Expression of the kallikrein gene family in normal and Alzheimer's disease brain. Neuroreport 12(12):2747-2751. DOI:10.1097/00001756-200108280-00031

47. Eissa A, Cretu D, Soosaipillai A, Thavaneswaran A, Pellett F, Diamandis A et al (2013) Serum kallikrein-8 correlates with skin activity, but not psoriatic arthritis, in patients with psoriatic disease. Clin Chem Lab Med 51:317-325. DOI:10.1515/cclm-2012-0251

48. Liu X, Quan B, Tian Z, Xi H, Jia G, Wang H et al (2017) Elevated expression of KLK8 predicts poor prognosis in colorectal cancer. Biomed Pharmacother 88:595-602.

DOI:10.1016/j.biopha.2017.01.112

49. Wang JP, Hielscher A. Fibronectin (2017) How its aberrant Expression in tumors may improve therapeutic targeting. J Cancer 8(4):674-682. DOI:10.7150/jca.16901

50. Tanjore $H$, Kalluri $R$ (2006) The role of type IV collagen and basement membranes in cancer progression and metastasis. Am J Pathol 168(3):715-717. DOI:10.2353/ajpath.2006.051321

51. Petraki C, Youssef YM, Dubinski W, Lichner Z, Scorilas A, Pasic MD et al (2012) Evaluation and prognostic significance of human tissue kallikrein-related peptidase 10 (KLK10) in colorectal cancer. Tumour Biol 33(4):1209-1214. DOI:10.1007/s13277-012-0368-5 
52. Li L, Xu N, Fan N, Meng Q, Luo W, Lv L et al (2015) Upregulated KLK10 inhibits esophageal cancer proliferation and enhances cisplatin sensitivity in vitro. Oncol Rep 34(5):2325-2332. DOI:10.3892/or.2015.4211

53. Tang L, Long Z, Feng G, Guo X, Yu M (2017) NES1/KLK10 promotes trastuzumab resistance via activation of PI3K/AKT signaling pathway in gastric cancer. J Cell Biochem 119(8):6398-6407. DOI:10.1002/jcb.26562

54. Xu Z, Chi P, Pan J, Shen S, Sun Y, Wang X et al (2016) Knockdown of KLK11 inhibits cell proliferation and increases oxaliplatin sensitivity in human colorectal cancer. Exp Ther Med 12(5):2855-2860. DOI:10.3892/etm.2016.3723

55. Zhang Y, Xu Z, Sun Y, Chi P, Lu X (2018) Knockdown of KLK11 reverses oxaliplatin resistance by inhibiting proliferation and activating apoptosis via suppressing the PI3K/AKT signal pathway in colorectal cancer cell. OncoTargets Ther 11:809. DOI:10.2147/OTT.S151867

56. Liu P, Cheng H, Roberts TM, Zhao JJ (2009) Targeting the phosphoinositide 3-kinase pathway in cancer. Nat Rev Drug Discov 8:627-644. DOI:10.1038/nrd2926

57. Le Roith D, Roberts CT. The insulin-like growth factor system and cancer. Cancer Lett. 2003; 195:127 - 37; DOI: 10.1016/s0304-3835(03)00159-9

58. Lawrence MG, Lai J, Clements JA (2010) Kallikreins on steroids: structure, function, and hormonal regulation of prostate-specific antigen and the extended kallikrein locus. Endocr Rev 31:407-446. DOI:10.1210/er.2009-0034

59. Luo LY, Grass L, Diamandis EP (2003) Steroid hormone regulation of the human kallikrein 10 (KLK10) gene in cancer cell lines and functional characterization of the KLK10 gene promoter. Clin Chim Acta 337:115-126. DOI:10.1016/j.cccn.2003.07.008

60. Paliouras M, Diamandis EP (2007) Coordinated steroid hormone-dependent and independent expression of multiple kallikreins in breast cancer cell lines. Breast Cancer Res Treat 102:7-18. DOI:10.1007/s10549-006-9312-y

61. Paliouras M, Diamandis EP (2008) Androgens act synergistically to enhance estrogen-induced upregulation of human tissue kallikreins 10,11 , and 14 in breast cancer cells via a membrane bound androgen receptor. Mol Oncol 4:413-424. DOI:10.1016/j.molonc.2008.01.001

62. Watrowski R, Castillo-Tong DC, Obermayr E, Zeillinger R (2020) Gene expression of kallikreins in breast cancer cell lines. Anticancer Res 40:2487-2495. DOI:10.21873/anticanres.14219

63. Shaw JL, Diamandis EP (2008) Regulation of human tissue kallikrein-related peptidase expression by steroid hormones in 32 cell lines. Biol Chem 389:1409-1419. DOl:10.1515/bc.2008.158

64. Shaw JL, Petraki C, Watson C, Bocking A, Diamandis EP (2008) Role of tissue kallikrein-related peptidases in cervical muscus remodeling and host defense. Biol Chem 389:1513-1522. DOI:10.1515/BC.2008.171

65. Jin H, Nagai N, Shigemasa K, Gu L, Tanimoto H, Yunokawa M et al (2006) Expression of tumorassociated differentially expressed Gene-14 (TADG-14/KLK8) and its protein hK8 in uterine endometria and endometrial carcinomas. Tumour Biol 27:274-282. DOI:10.1159/000094741 
66. Pasic MD, Olkhov E, Bapat B, Yousef GM (2012) Epigenetic regulation of kallikrein-related peptidases: there is a whole new world out there. Biol Chem 393:319-330. DOl:10.1515/hsz-2011-0273

67. Gucalp A, Traina TA (2017) The Androgen Receptor: Is It a Promising Target? Ann Surg Oncol 24(10):2876-2880. DOI:10.1245/s10434-017-5961-9

68. Prassas I, Eissa A, Poda G, Diamandis EP (2015) Unleashing the therapeutic potential of human kallikrein-related serine proteases. Nat Rev Drug Discov 14(3):183-202. DOI:10.1038/nrd4534

69. Eissa A, Amodeo V, Smith CR, Diamandis EP (2011) Kallikrein-related peptidase-8 (KLK8) is an active serine protease in human epidermis and sweat and is involved in a skin barrier proteolytic cascade. $J$ Biol Chem 286:687-706. DOI:10.1074/jbc.M110.125310

70. Yoon H, Laxmikanthan G, Lee J, Blaber SI, Rodriguez A, Kogot JM et al (2007) Activation profiles and regulatory cascades of the human kallikrein-related peptidases. J Biol Chem 282(44):31852-31864. DOI:10.1074/jbc.M705190200

71. Yoon H, Blaber SI, Li W, Scarisbrick IA, Blaber M (2013) Activation profiles of human kallikrein-related peptidases by matrix metalloproteinases. Biol Chem 394(1):137-147. DOI:10.1515/hsz-2012-0249

\section{Figures}



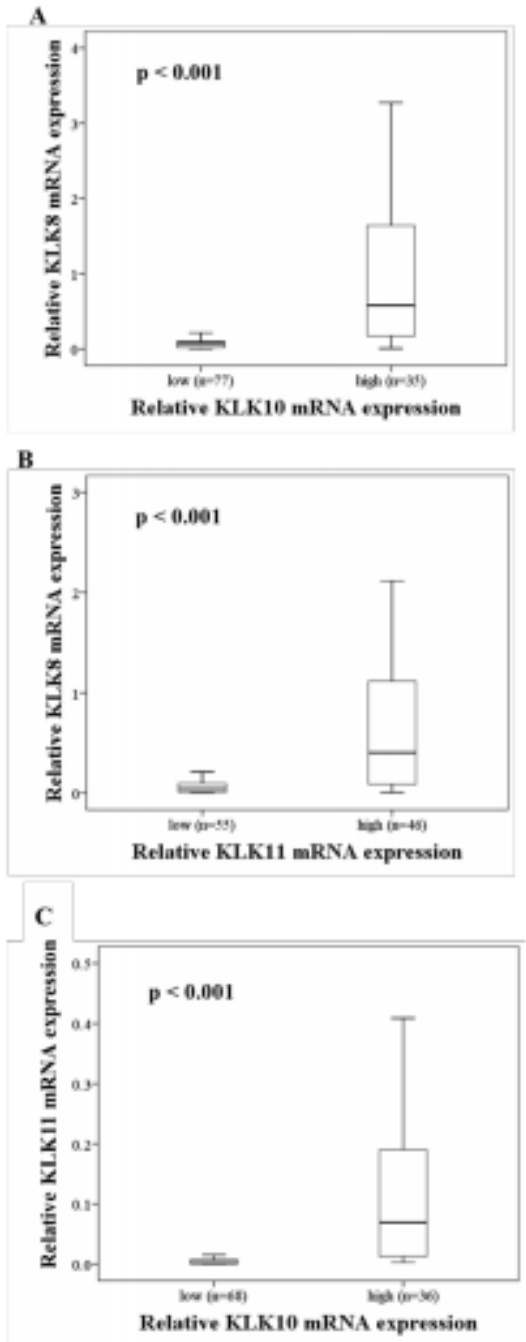

\section{Figure 1}

Correlation of KLKs mRNA expression in tumor specimens of triple-negative breast cancer patients. By applying the Mann-Whitney test, KLK8 mRNA expression levels were found to be significantly correlated with KLK10 mRNA expression levels $(A, r s=0.612, p<0.001)$. Similarly, a significantly positive correlation was also observed between KLK8 and KLK11 (B, rs $=0.460, p<0.001)$ and between KLK10 and KLK11 (C, $r s=0.722, p<0.001)$. 

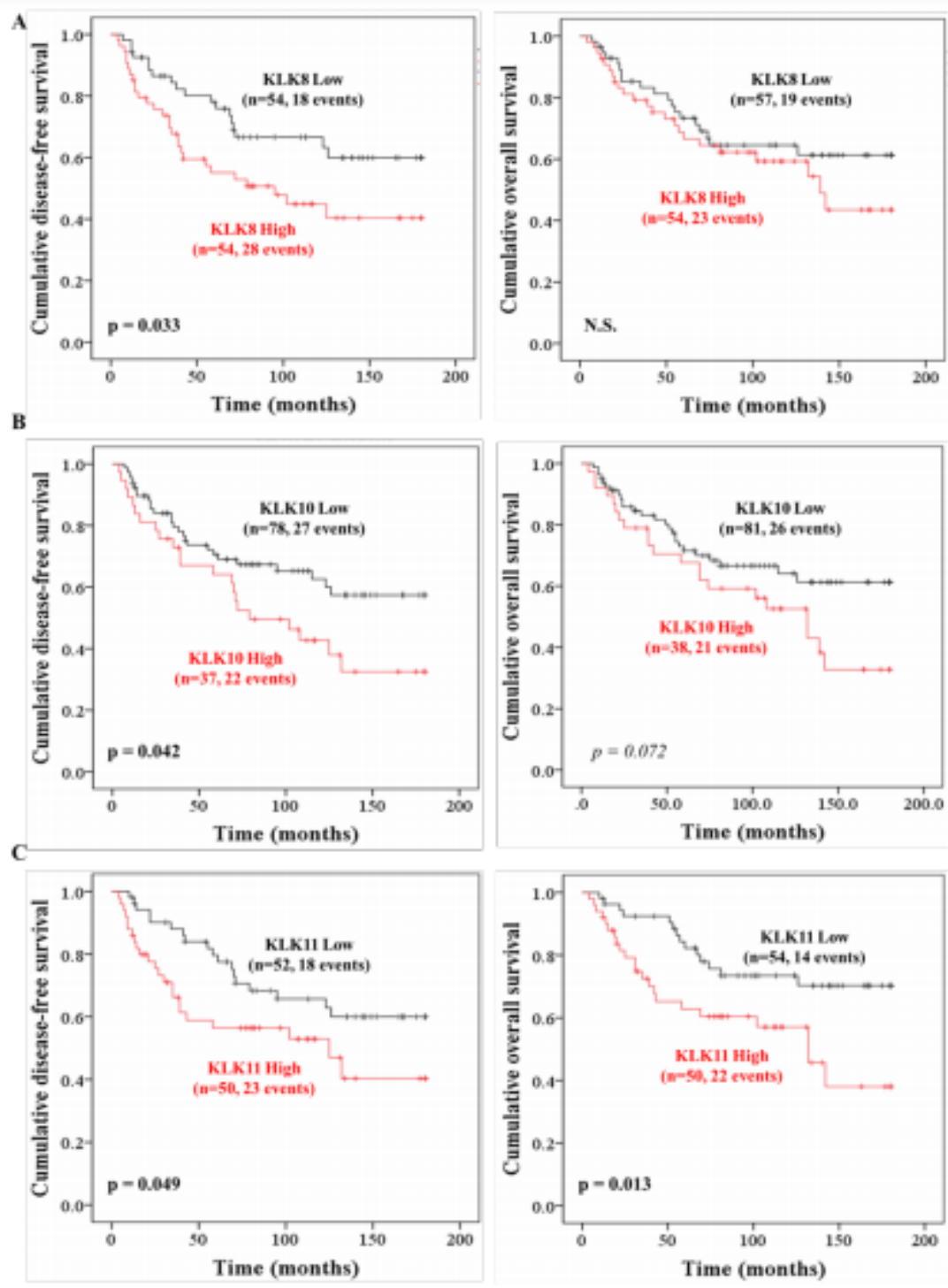

Figure 2

Probability of disease-free survival (DFS) and overall survival (OS) of patients with triple-negative breast cancer as stratified by KLK8, KLK10 and KLK11 mRNA expression levels, respectively, in primary tumor tissues. Patients with elevated KLK8 (A) and KLK10 (B) mRNA expression levels show significantly shortened DFS (Kaplan-Meier analysis, $p=0.033, p=0.042$, respectively), while patients with high mRNA levels of KLK11 (C) display significantly poor DFS ( $p=0.049)$ as well as OS $(p=0.013)$, compared to those with low mRNA expression levels. 
A

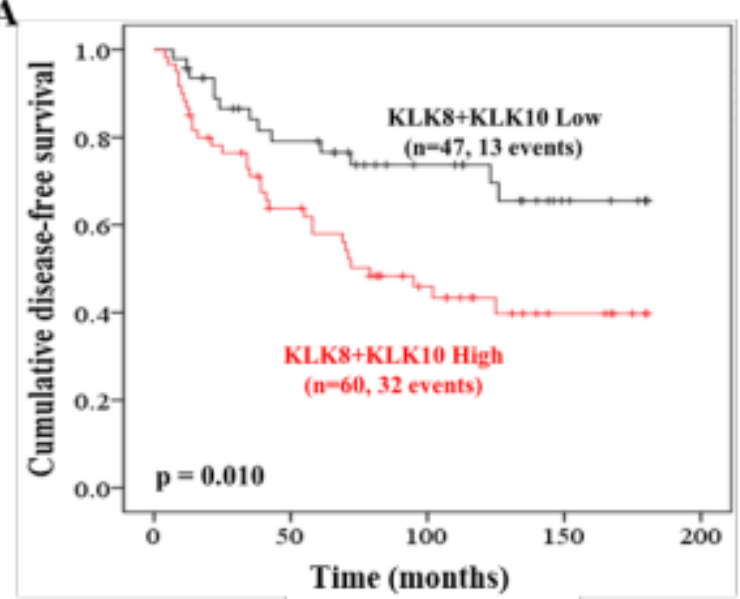

C

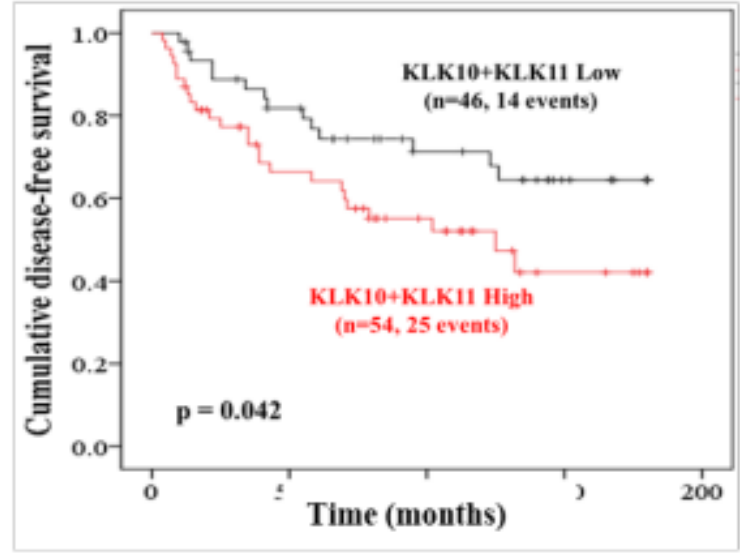

B

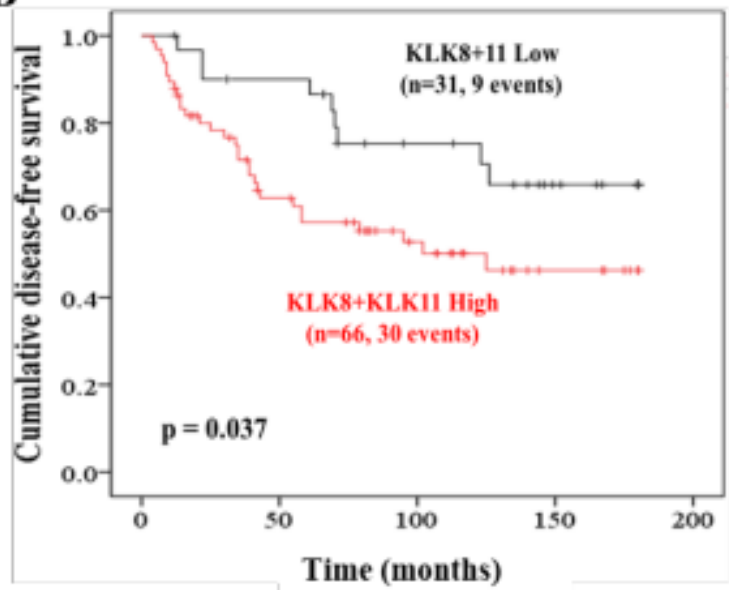

D

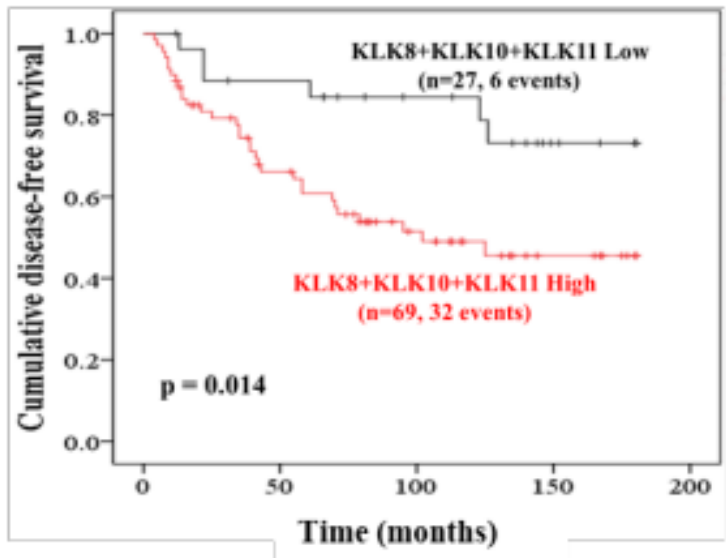

\section{Figure 3}

Probability of disease-free survival (DFS) of patients with triple-negative breast cancer as stratified by KLK8+KLK10, KLK8+KLK11, KLK10+KLK11, and KLK8+KLK10+KLK11 mRNA expression levels, respectively, in primary tumor tissues. (A) KLK8+KLK10 mRNA expression (low/low versus high and/or high); (B) KLK8+KLK11 mRNA expression (low/low versus high and/or high); (C) KLK10+KLK11 mRNA expression (low/low versus high and/or high); (D) KLK8+KLK10+KLK11 mRNA expression (low/low/low versus high and/or high and/or high).

\section{Supplementary Files}

This is a list of supplementary files associated with this preprint. Click to download.

- SupplFig1YLiu.pdf

- SuppIFig2YLiu.pdf

- SupplTab1YLiu.pdf 